Article

\title{
Eigenvalue Problem for Discrete Jacobi-Sobolev Orthogonal Polynomials
}

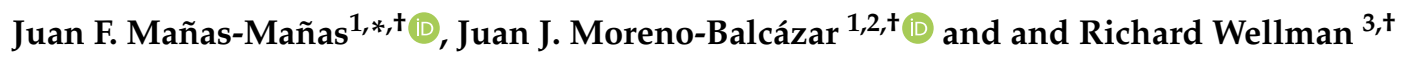 \\ 1 Departamento de Matemáticas, Universidad de Almería, 04120 Almería, Spain; balcazar@ual.es \\ 2 Instituto Carlos I de Física Teórica y Computacional, 18071 Granada, Spain \\ 3 Department of Mathematics \& Computer Science, Colorado College, CO 80903, USA; \\ rwellman@coloradocollege.edu \\ * Correspondence: jmm939@ual.es; Tel.: +34-950015813 \\ + These authors contributed equally to this work.
}

Received: 29 October 2019; Accepted: 22 January 2020; Published: 3 February 2020

check for updates

\begin{abstract}
In this paper, we consider a discrete Sobolev inner product involving the Jacobi weight with a twofold objective. On the one hand, since the orthonormal polynomials with respect to this inner product are eigenfunctions of a certain differential operator, we are interested in the corresponding eigenvalues, more exactly, in their asymptotic behavior. Thus, we can determine a limit value which links this asymptotic behavior and the uniform norm of the orthonormal polynomials in a logarithmic scale. This value appears in the theory of reproducing kernel Hilbert spaces. On the other hand, we tackle a more general case than the one considered in the literature previously.
\end{abstract}

Keywords: Sobolev orthogonal polynomials; Jacobi weight; asymptotics

MSC: 33C47; 34L15; 42C05

\section{Introduction}

The study of Sobolev inner products and the properties of the corresponding orthogonal polynomials is a relevant topic within the theory of orthogonal polynomials. Indeed, Sobolev orthogonality is a recent topic in the theory of orthogonal polynomials. In fact, it has been developed throughout the last 30 years, although the first seminal paper was written by Lewis in 1947 (see [1]) in the framework of the simultaneous approximation to a function and its derivatives in the corresponding Sobolev space. The relevance of Sobolev orthogonal polynomials (SOP) is given by the fact that they are not orthogonal in a standard way. Thus, if we consider a typical Sobolev inner product in the appropriate Sobolev space, then some nice properties of standard orthogonal polynomials (such as the three-term recurrence relation and the interlacing properties of zeros) are lost. Furthermore, some powerful methods and techniques developed for standard orthogonal polynomials have not found their equivalence in SOP and many questions remain unanswered. Therefore, we need to construct new techniques to obtain algebraic, differential, and asymptotic properties of the SOP. This fact was already noticed on the first papers by German mathematicians such as in [2,3], or [4].

Concretely, given a Sobolev inner product (SIP)

$$
(f, g)=\int_{\mathbb{R}} f(x) g(x) d \mu_{0}+\sum_{k=1}^{m} \int_{\mathbb{R}} f^{(k)}(x) g^{(k)}(x) d \mu_{k}
$$


in the Sobolev space

$$
W^{2, m}\left(\mu_{0}, \mu_{1}, \ldots, \mu_{m}\right):=\left\{f: \int_{\mathbb{R}}|f|^{2} d \mu_{0}+\sum_{k=1}^{m} \int_{\mathbb{R}}\left|f^{(k)}\right|^{2} d \mu_{k}<+\infty\right\},
$$

where $\mu_{i}$ with $i=0, \ldots, m$ are positive Borel measures supported on the real line, we usually classify this inner product as follows (see, for example, [5]):

- $\quad$ Continuous SIP. All the measures $\mu_{i}$, with $i=0, \ldots, m$, have continuous support.

- Discrete SIP. The measure $\mu_{0}$ has continuous support and the measures $\mu_{1}, \ldots, \mu_{m}$ are discrete, i.e., they are supported on finite subsets.

- Discrete-Continuous SIP. The measure $\mu_{m}$ has continuous support and the measures $\mu_{0}, \ldots, \mu_{m-1}$ are discrete.

Obviously, when $m \geq 1$, we have $(x f, g) \neq(f, x g)$. As it is well known, the identity $(x f, g)=$ $(f, x g)$ is the key to obtain many properties of standard orthogonal polynomials, therefore the study of Sobolev orthogonal polynomials needs a different approach as we have mentioned previously.

In the first few years, the most exhaustively studied case in the literature corresponded to $m=1$. Thus, in the continuous case, a breaking paper was given in [6] where the concept of a pair of coherent measures was introduced. This concept was originally useful for minimizing the Gibbs phenomenon that occurs when approximating a function by the partial sum of a Fourier series (see [7]). Later, it has been very fruitful in the study of algebraic and asymptotic properties of Sobolev orthogonal polynomials. In fact, the original concept was generalized on several occasions (for example, in [8] or [9]), being the most general the one introduced in 2014 with the $(M, N)$-coherent pairs of order $(m, k)$ (see [10]).

On the other hand, the discrete-continuous case attracted interest when it was discovered that this type of inner product provides Sobolev orthogonality to families of classical orthogonal polynomials with nonstandard parameters (Gegenabauer, Jacobi, and Laguerre polynomials), see details in the papers [11-15] or in a more general framework [16].

The discrete case has been studied extensively since the 1990s, with very general papers being [17] some of the most relevant. Along these years, algebraic, asymptotic, and differential properties of the corresponding orthogonal polynomials have been studied. The motivation of this paper is to tackle one aspect less studied in the literature. Concretely, it is known from some papers by Bavinck in the 1980s that certain families of discrete Sobolev orthogonal polynomials are eigenfunctions of a differential operator. In this way, we are interested in knowing the asymptotic behavior of the corresponding eigenvalues, which allows us to get a limit value of interest in the theory of reproducing kernel Hilbert spaces.

The chronology of the advances in the theory of the Sobolev orthogonality has been collected in several surveys: [5,18-22]. Even a very brief outreach paper has been published-see [23]. About the applications of SOP, we have already mentioned the usefulness of these nonstandard polynomials in the simultaneous approximation of a function and their derivatives as well as to tackle the Gibbs phenomenon, together with the fact that standard polynomials with nonstandard parameters are orthogonal with respect to a SIP. More recently, other different applications have been obtained among others in [24-26] or [27] related to use SOP for solving some type of Cauchy problem for ordinary differential equations.

In this paper, we focus our attention on a special case of the discrete SIP

$$
(f, g)_{S}=\int f(x) g(x) d \mu+\sum_{i=0}^{p} M_{i} f^{(i)}(c) g^{(i)}(c), \quad M_{i} \geq 0, i=0, \ldots, p-1, M_{p}>0,
$$

where $\mu$ is a positive Borel measure. The study of the orthogonal polynomials with respect to the above inner product has attracted the attention of researchers in recent decades (see [5] and 
the aforementioned surveys). If we treat these polynomials as eigenfunctions of a differential operator, the study of the corresponding eigenvalues arises as a topic of interest. Thus, very recently, the asymptotic behavior of the eigenvalues has been studied in [28] when $\mu$ is a classical continuous measure (Jacobi, Laguerre, or Hermite) and $c$ is a point located on the real axis adequately.

Here, we consider a discrete Sobolev inner product involving the Jacobi weight and including two derivatives of different order located at -1 and 1 . Concretely,

$$
(f, g)_{S}=\int_{-1}^{1} f(x) g(x)(1-x)^{\alpha}(1+x)^{\beta} d x+M f^{\left(j_{1}\right)}(1) g^{\left(j_{1}\right)}(1)+N f^{\left(j_{2}\right)}(-1) g^{\left(j_{2}\right)}(-1)
$$

where $M, N>0, \alpha, \beta>-1$, and $j_{1}, j_{2} \in \mathbb{N} \cup\{0\}$. Let $\left\{q_{n}^{(M, N)}\right\}_{n \geq 0}$ be the sequence of orthonormal polynomials with respect to (1). It is well known that these polynomials are eigenfunctions of a differential operator $\mathcal{T}$ (see [29]). Thus, our first goal is to establish the asymptotic behavior of the corresponding eigenvalues which we denote by $\varrho_{n}$ and as an application of this result and the properties of the corresponding Sobolev orthonormal polynomials, we can compute the value

$$
r_{0}=\limsup _{n \rightarrow+\infty} \frac{\log \left(n \max _{x \in[-1,1]}\left|q_{n}^{(M, N)}(x)\right|^{2}\right)}{\log \left(\varrho_{n}\right)} .
$$

This value is relevant for the work that one of the authors of this article is developing in the framework of reproducing kernel Hilbert spaces and their interactions with the applied machine learning [30].

We will prove that in our case it is enough to compute the value

$$
s_{0}=\lim _{n \rightarrow+\infty} \frac{\log \left(\max _{x \in[-1,1]}\left|q_{n}^{(M, N)}(x)\right|\right)}{\log \left(\varrho_{n}\right)},
$$

to obtain the value $r_{0}$. This value $s_{0}$ was already computed in the particular case of the symmetric case in [31], that is, when we consider $\alpha=\beta, M=N$, and $j_{1}=j_{2}$ (Gegenbauer case). The symmetry provides us with relations (see e.g., ([31], Proposition 5)) that we have not been able to derive for this nonsymmetric case.

The structure of the paper is the following: in Section 2, basic properties of Jacobi polynomials are introduced; in Section 3, the asymptotic behavior of the eigenvalues $\varrho_{n}$ is obtained. Section 4 is devoted to obtaining algebraic, differential, and asymptotic properties of these discrete Sobolev orthogonal polynomials, which, together with the results in Section 3, allow us to get the value $r_{0}$ in Section 5 . Finally, we have included Section 6 with the conclusions and some open problems.

\section{Background on Jacobi Orthogonal Polynomials}

We denote by $P_{n}^{(\alpha, \beta)}(x)$ the classical Jacobi orthogonal polynomials with respect to the weight function $(1-x)^{\alpha}(1+x)^{\beta}$, on $(-1,1)$ with $\alpha, \beta>-1$ and taking the standardization given in ([32], f. (4.1.1)):

$$
P_{n}^{(\alpha, \beta)}(1)=\left(\begin{array}{c}
n+\alpha \\
n
\end{array}\right)=\frac{\Gamma(n+\alpha+1)}{\Gamma(n+1) \Gamma(\alpha+1)} .
$$

Using ([32], f. (4.1.4)), we have

$$
P_{n}^{(\alpha, \beta)}(-1)=(-1)^{n} P_{n}^{(\beta, \alpha)}(1)=(-1)^{n}\left(\begin{array}{c}
n+\beta \\
n
\end{array}\right)=(-1)^{n} \frac{\Gamma(n+\beta+1)}{\Gamma(n+1) \Gamma(\beta+1)}
$$


Their derivatives satisfy (see, ([33], f. (4)) and ([32], f. (4.21.7)))

$$
\left(P_{n}^{(\alpha, \beta)}\right)^{(k)}(x)=\frac{1}{2^{k}} \frac{\Gamma(n+\alpha+\beta+k+1)}{\Gamma(n+\alpha+\beta+1)} P_{n-k}^{(\alpha+k, \beta+k)}(x), \quad k \geq 0 .
$$

Using (2) and (4), we deduce

$$
\left(P_{n}^{(\alpha, \beta)}\right)^{(k)}(1)=\frac{1}{2^{k}} \frac{\Gamma(n+\alpha+\beta+k+1)}{\Gamma(n+\alpha+\beta+1)} \frac{\Gamma(n+\alpha+1)}{\Gamma(n-k+1) \Gamma(\alpha+k+1)},
$$

where $\left(P_{n}^{(\alpha, \beta)}\right)^{(k)}(1)$ denotes the $k$-th derivative of $P_{n}^{(\alpha, \beta)}(x)$ evaluated at $x=1$. Thus, using (3) and (4) we have:

$$
\left(P_{n}^{(\alpha, \beta)}\right)^{(k)}(-1)=(-1)^{n-k} \frac{1}{2^{k}} \frac{\Gamma(n+\alpha+\beta+k+1)}{\Gamma(n+\alpha+\beta+1)} \frac{\Gamma(n+\beta+1)}{\Gamma(n-k+1) \Gamma(\beta+k+1)} .
$$

The norm of these polynomials is given by (see, ([32], f. (4.3.3))):

$$
\left\|P_{n}^{(\alpha, \beta)}\right\|^{2}=\frac{2^{\alpha+\beta+1}}{2 n+\alpha+\beta+1} \frac{\Gamma(n+\alpha+1) \Gamma(n+\beta+1)}{\Gamma(n+1) \Gamma(n+\alpha+\beta+1)} .
$$

Finally, they satisfy the second-order differential equation (see ([32], f. (4.2.1))):

$$
\left(x^{2}-1\right) y^{\prime \prime}(x)+(\alpha-\beta+(\alpha+\beta+2) x) y^{\prime}(x)=n(n+\alpha+\beta+1) y(x),
$$

i.e., $P_{n}^{(\alpha, \beta)}$ are the eigenfunctions of the differential operator

$$
\mathcal{J}:\left(x^{2}-1\right) \mathcal{D}^{2}+(\alpha-\beta+(\alpha+\beta+2) x) \mathcal{D},
$$

where $\mathcal{D}$ denotes the derivative operator. Thus, we can write down

$$
\mathcal{J} P_{n}^{(\alpha, \beta)}(x)=n(n+\alpha+\beta+1) P_{n}^{(\alpha, \beta)}(x),
$$

so

$$
\lambda_{n}=n(n+\alpha+\beta+1)
$$

are the corresponding eigenvalues.

Along the paper, we use the well-known Stirling's formula (see, for example, ([34], f. (5.11.13))):

$$
\lim _{n \rightarrow+\infty} \frac{n^{b-a} \Gamma(n+a)}{\Gamma(n+b)}=1 .
$$

Next, we give some asymptotic behaviors which will be useful in the next section.

Lemma 1. Letting $k$ be a nonnegative integer, we have:

$$
\begin{aligned}
\lim _{n \rightarrow+\infty} \frac{\left(P_{n}^{(\alpha, \beta)}\right)^{(k)}(1)}{n^{\alpha+2 k}} & =\frac{1}{2^{k} \Gamma(\alpha+k+1)}, \\
\lim _{n \rightarrow+\infty} \frac{(-1)^{n}\left(P_{n}^{(\alpha, \beta)}\right)^{(k)}(-1)}{n^{\beta+2 k}} & =\frac{(-1)^{k}}{2^{k} \Gamma(\beta+k+1)} .
\end{aligned}
$$

In addition,

$$
\lim _{n \rightarrow+\infty} n\left\|P_{n}^{(\alpha, \beta)}\right\|^{2}=2^{\alpha+\beta} .
$$


Proof. We can deduce them straightforwardly using (5)-(7) and (9).

The kernel polynomials and their derivatives are given by

$$
K_{n}^{(j, k)}(x, y)=\sum_{i=0}^{n} \frac{\left(P_{i}^{(\alpha, \beta)}\right)^{(j)}(x)\left(P_{i}^{(\alpha, \beta)}\right)^{(k)}(y)}{\left\|P_{i}^{(\alpha, \beta)}\right\|^{2}}, \quad j, k \in \mathbb{N} \cup\{0\} .
$$

For our purposes, we need to know some asymptotic behaviors of these kernel polynomials evaluated at the points 1 and -1 . Next, we introduce a result which generalizes some particular cases obtained in ([33], Lemma 1) or ([35], Pag. 147).

Proposition 1. Let $j, k$ be nonnegative integers. Then, we have

$$
\begin{gathered}
\lim _{n \rightarrow+\infty} \frac{K_{n-1}^{(j, k)}(1,1)}{n^{2 \alpha+2 j+2 k+2}}=\frac{1}{2^{\alpha+\beta+j+k+1}(\alpha+j+k+1) \Gamma(\alpha+j+1) \Gamma(\alpha+k+1)} . \\
\lim _{n \rightarrow+\infty} \frac{K_{n-1}^{(j, k)}(-1,-1)}{n^{2 \beta+2 j+2 k+2}}=\frac{(-1)^{j+k}}{2^{\alpha+\beta+j+k+1}(\beta+j+k+1) \Gamma(\beta+j+1) \Gamma(\beta+k+1)} . \\
\lim _{n \rightarrow+\infty} \frac{(-1)^{n} K_{n}^{(j, k)}(1,-1)}{n^{\alpha+\beta+2 j+2 k+1}}=\frac{(-1)^{k}}{2^{\alpha+\beta+j+k+1} \Gamma(\alpha+j+1) \Gamma(\beta+k+1)} .
\end{gathered}
$$

Proof. To prove these results, we use (2)-(7), (9), the Stolz-Cesàro criterion, and the following limit

$$
\lim _{n \rightarrow+\infty} \frac{n^{\alpha+\beta+2 j+2 k+2}-(n-1)^{\alpha+\beta+2 j+2 k+2}}{(\alpha+\beta+2 j+2 k+2) n^{\alpha+\beta+2 j+2 k+1}}=1 .
$$

(13a) This case has been proved in ([33], Lemma 1).

(13b) We use the same technique as in ([33], Lemma 1), getting

$$
\begin{aligned}
\lim _{n \rightarrow+\infty} \frac{K_{n-1}^{(j, k)}(-1,-1)}{n^{2 \beta+2 j+2 k+2}} & =\lim _{n \rightarrow+\infty} \frac{K_{n-1}^{(j, k)}(-1,-1)-K_{n-2}^{(j, k)}(-1,-1)}{n^{2 \beta+2 j+2 k+2}-(n-1)^{2 \beta+2 j+2 k+2}} \\
& =\lim _{n \rightarrow+\infty} \frac{\left(P_{n-1}^{(\alpha, \beta)}\right)^{(j)}(-1)\left(P_{n-1}^{(\alpha, \beta)}\right)^{(k)}(-1)}{\left.(2 \beta+2 j+2 k+2)\left\|P_{n-1}^{(\alpha, \beta)}\right\|\right|^{2} n^{2 \beta+2 j+2 k+1}} \\
& =\frac{1}{2^{\alpha+\beta}(2 \beta+2 j+2 k+2)} \frac{(-1)^{j}}{2^{j} \Gamma(\beta+j+1)} \frac{(-1)^{k}}{2^{k} \Gamma(\beta+k+1)}
\end{aligned}
$$

(13c) In this case, we cannot use the Stolz-Cesàro criterion in the previous straightforward way. Then, we use a different approach based on the Christoffel-Darboux formula (see ([32], f. (4.5.2))):

$$
K_{n}^{(0,0)}(x, y)=a_{n} \frac{P_{n+1}^{(\alpha, \beta)}(x) P_{n}^{(\alpha, \beta)}(y)-P_{n}^{(\alpha, \beta)}(x) P_{n+1}^{(\alpha, \beta)}(y)}{x-y}
$$

with $a_{n}=\frac{2^{-\alpha-\beta}}{2 n+\alpha+\beta+2} \frac{\Gamma(n+2) \Gamma(n+\alpha+\beta+2)}{\Gamma(n+\alpha+1) \Gamma(n+\beta+1)}$.

First, it is enough to apply (9) to get

$$
\lim _{n \rightarrow+\infty} \frac{a_{n}}{n}=\frac{1}{2^{\alpha+\beta+1}} .
$$


Now, using a Leibniz rule, we obtain (see also [36])

$$
\begin{aligned}
K_{n}^{(0, k)}(x, y) & =a_{n}\left[P_{n+1}^{(\alpha, \beta)}(x) \frac{\partial^{k}}{\partial y^{k}}\left(\frac{P_{n}^{(\alpha, \beta)}(y)}{x-y}\right)-P_{n}^{(\alpha, \beta)}(x) \frac{\partial^{k}}{\partial y^{k}}\left(\frac{P_{n+1}^{(\alpha, \beta)}(y)}{x-y}\right)\right] \\
& =a_{n}\left[P_{n+1}^{(\alpha, \beta)}(x)\left(\sum_{i=0}^{k} \frac{k !\left(P_{n}^{(\alpha, \beta)}\right)^{(i)}(y)}{i !(x-y)^{k-i+1}}\right)-P_{n}^{(\alpha, \beta)}(x)\left(\sum_{i=0}^{k} \frac{k !\left(P_{n+1}^{(\alpha, \beta)}\right)^{(i)}(y)}{i !(x-y)^{k-i+1}}\right)\right] .
\end{aligned}
$$

Taking derivatives again, we deduce

$$
\begin{aligned}
K_{n}^{(j, k)}(x, y)=a_{n} & {\left[\sum_{i=0}^{k}\left(P_{n}^{(\alpha, \beta)}\right)^{(i)}(y) \frac{k !}{i !} \frac{\partial^{j}}{\partial x^{j}}\left(\frac{P_{n+1}^{(\alpha, \beta)}(x)}{(x-y)^{k-i+1}}\right)\right.} \\
& \left.-\sum_{i=0}^{k}\left(P_{n+1}^{(\alpha, \beta)}\right)^{(i)}(y) \frac{k !}{i !} \frac{\partial^{j}}{\partial x^{j}}\left(\frac{P_{n}^{(\alpha, \beta)}(x)}{(x-y)^{k-i+1}}\right)\right] \\
=a_{n} & {\left[\sum_{i=0}^{k} \sum_{\ell=0}^{j} \frac{k !}{i !} \frac{\left(P_{n+1}^{(\alpha, \beta)}\right)^{(\ell)}(x)\left(P_{n}^{(\alpha, \beta)}\right)^{(i)}(y)}{(x-y)^{k-i+j-\ell+1}}\left(\begin{array}{l}
j \\
\ell
\end{array}\right) \prod_{q=0}^{j-\ell-1}(-k+i-1-q)\right.} \\
& -\sum_{i=0}^{k} \sum_{\ell=0}^{j} \frac{k !}{i !} \frac{\left(P_{n}^{(\alpha, \beta)}\right)^{(\ell)}(x)\left(P_{n+1}^{(\alpha, \beta)}\right)^{(i)}(y)}{(x-y)^{k-i+j-\ell+1}}\left(\begin{array}{l}
j \\
\ell
\end{array}\right)^{j-\ell-1} \prod_{q=0}^{(-k+k+i-1-q)] .}
\end{aligned}
$$

Dividing the previous expression by $(-1)^{n} n^{\alpha+\beta+2 j+2 k+1}$ and evaluating at $x=1$ and $y=-1$, we get

$$
\begin{aligned}
& \frac{(-1)^{n} K_{n}^{(j, k)}(1,-1)}{n^{\alpha+\beta+2 j+2 k+1}} \\
= & \frac{a_{n}}{n}\left[\sum_{i=0}^{k} \sum_{\ell=0}^{j} \frac{k !}{i !} \frac{\left(P_{n+1}^{(\alpha, \beta)}\right)^{(\ell)}(1)\left(P_{n}^{(\alpha, \beta)}\right)^{(i)}(-1)}{(-1)^{n} n^{\alpha+\beta+2 j+2 k} 2^{k-i+j-\ell+1}\left(\begin{array}{l}
j \\
\ell
\end{array}\right) \prod_{q=0}^{j-\ell-1}(-k+i-1-q)}\right. \\
& \left.\quad-\sum_{i=0}^{k} \sum_{\ell=0}^{j} \frac{k !}{i !} \frac{\left(P_{n}^{(\alpha, \beta)}\right)^{(\ell)}(1)\left(P_{n+1}^{(\alpha, \beta)}\right)^{(i)}(-1)}{(-1)^{n} n^{\alpha+\beta+2 j+2 k} 2^{k-i+j-\ell+1}}\left(\begin{array}{l}
j \\
\ell
\end{array}\right) \prod_{q=0}^{j-\ell-1}(-k+i-1-q)\right] .
\end{aligned}
$$

Finally, taking limits in (15) and considering (10), (11), and (14), all terms tend to zero except when $i=k$ and $\ell=j$, so

$$
\begin{aligned}
& \lim _{n \rightarrow+\infty} \frac{(-1)^{n} K_{n}^{(j, k)}(1,-1)}{n^{\alpha+\beta+2 j+2 k+1}} \\
= & \lim _{n \rightarrow+\infty} \frac{a_{n}}{n}\left[\frac{\left(P_{n+1}^{(\alpha, \beta)}\right)^{(j)}(1)(-1)^{n}\left(P_{n}^{(\alpha, \beta)}\right)^{(k)}(-1)}{2 n^{\alpha+\beta+2 j+2 k}}+\frac{\left(P_{n}^{(\alpha, \beta)}\right)^{(j)}(1)(-1)^{n+1}\left(P_{n+1}^{(\alpha, \beta)}\right)^{(k)}(-1)}{2 n^{\alpha+\beta+2 j+2 k}}\right] \\
= & \frac{1}{2^{\alpha+\beta+1}} \frac{1}{2}\left(\frac{1}{2^{j} \Gamma(\alpha+j+1)} \frac{(-1)^{k}}{2^{k} \Gamma(\beta+k+1)}+\frac{1}{2^{j} \Gamma(\alpha+j+1)} \frac{(-1)^{k}}{2^{k} \Gamma(\beta+k+1)}\right) \\
= & \frac{(-1)^{k}}{2^{\alpha+\beta+j+k+1} \Gamma(\alpha+j+1) \Gamma(\beta+k+1)} .
\end{aligned}
$$




\section{Asymptotic Behavior of the Eigenvalues $\varrho_{n}$}

In [29], it was established that the discrete Sobolev orthonormal polynomials $q_{n}^{(M, N)}$ are eigenfunctions of a certain differential operator $\mathcal{T}$, i.e., $\mathcal{T} q_{n}^{(M, N)}=\varrho_{n} q_{n}^{(M, N)}$. In the same paper, see ([29], f. (5)), and, under certain conditions that we will expose later, the author claims

$$
\varrho_{n}=\lambda_{n}+M \alpha_{n}+N \beta_{n}+M N \gamma_{n}, \quad n \geq \max \left\{j_{1}, j_{2}\right\},
$$

where $\lambda_{n}$ is defined in (8) and

$$
\begin{aligned}
& \alpha_{n}=\alpha_{j_{1}}+\sum_{i=j_{1}+1}^{n}\left(\lambda_{i}-\lambda_{i-1}\right) K_{i-1}^{\left(j_{1}, j_{1}\right)}(1,1), \\
& \beta_{n}=\beta_{j_{2}}+\sum_{i=j_{2}+1}^{n}\left(\lambda_{i}-\lambda_{i-1}\right) K_{i-1}^{\left(j_{2}, j_{2}\right)}(-1,-1) \\
& \gamma_{n}=\gamma_{\max \left\{j_{1}, j_{2}\right\}}+\sum_{i=\max \left\{j_{1}, j_{2}\right\}+1}^{n}\left(\lambda_{i}-\lambda_{i-1}\right)\left[K_{i-1}^{\left(j_{1}, j_{1}\right)}(1,1) K_{i-1}^{\left(j_{2}, j_{2}\right)}(-1,-1)-\left(K_{i-1}^{\left(j_{1}, j_{2}\right)}(1,-1)\right)^{2}\right] .
\end{aligned}
$$

Moreover, the author proved that there are two ways to construct the differential operator $\mathcal{T}$. These two ways depend on the relation between $j_{1}, j_{2}$ (either $j_{1} \leq j_{2}$ or $j_{2} \leq j_{1}$ ), but in both cases the differential operator obtained is the same, so the expression (16) for the eigenvalues holds in both cases (for more details, see [29]).

However, when the inner product (1) is symmetric, i.e., when $\alpha=\beta, M=N$, and $j_{1}=j_{2}$, then

$$
\varrho_{n}=\lambda_{n}+M \mu_{n}
$$

and the process to establish the asymptotic behavior of $\varrho_{n}$ is technically easier. Thus, through this paper, we assume that the inner product (1) is nonsymmetric. The results corresponding to the symmetric case can be found in [31].

For convenience, we assume $j_{1} \leq j_{2}$. Denote by $s_{n}^{(M)}(x)$ the orthogonal polynomials with respect to the inner product

$$
(f, g)=\int_{-1}^{1} f(x) g(x)(1-x)^{\alpha}(1+x)^{\beta} d x+M f^{\left(j_{1}\right)}(1) g^{\left(j_{1}\right)}(1) .
$$

In this case, the condition to obtain $(16)$ is $\left(s_{n}^{(M)}\right)^{\left(j_{2}\right)}(-1) \neq 0$ while $j_{2}<n$. Using ([33], Prop. 2), we can assure that $s_{n}^{(M)}(x)$ has $n$ real and simple zeros, and they are located in $(-1,1+\varepsilon]$ with $\varepsilon \geq 0$. On the other hand, using Rolle's Theorem, between two zeros of $s_{n}^{(M)}(x)$, one zero of $\left(s_{n}^{(M)}\right)^{\prime}(x)$ exists, so we can assure that there are $n-1$ real and simple zeros of $\left(s_{n}^{(M)}\right)^{\prime}(x)$ in $(-1,1+\varepsilon)$. However, $\left(s_{n}^{(M)}\right)^{\prime}(x)$ is a polynomial of degree $n-1$, so it has $n-1$ zeros, thus $\left(s_{n}^{(M)}\right)^{\prime}(-1) \neq 0$. We can repeat this process and we can assure that $\left(s_{n}^{(M)}\right)^{\left(j_{2}\right)}(-1) \neq 0$, with $j_{2}<n$. For the case $j_{1} \geq j_{2}$, we can proceed in a similar way (see also [36]).

Following [29], $\alpha_{0}=0$ and $\left\{\alpha_{i}\right\}_{i=1}^{j_{1}}$ can be chosen arbitrarily. Thus, we take $\alpha_{1}=\alpha_{2}=\cdots=$ $\alpha_{j_{1}}=0$. Similarly for the values $\beta_{n}$ and $\gamma_{n}$, it is necessary that $\beta_{0}=\gamma_{0}=0$ and the quantities $\left\{\beta_{i}\right\}_{i=1}^{j_{2}}$ and $\left\{\gamma_{i}\right\}_{i=1}^{\max \left\{j_{1}, j_{2}\right\}}$ can be chosen arbitrarily. Again, we choose $\beta_{1}=\beta_{2}=\cdots=\beta_{j_{2}}=0$ and $\gamma_{1}=\gamma_{2}=\cdots=\gamma_{\max \left\{j_{1}, j_{2}\right\}}=0$. Therefore, taking into account

$$
\lambda_{n}-\lambda_{n-1}=2 n+\alpha+\beta,
$$

and substituting in (16), the expressions $\alpha_{n}, \beta_{n}$ and $\gamma_{n}$ can be simplified to 


$$
\begin{aligned}
& \alpha_{n}=\sum_{i=j_{1}+1}^{n}(2 i+\alpha+\beta) K_{i-1}^{\left(j_{1}, j_{1}\right)}(1,1), \\
& \beta_{n}=\sum_{i=j_{2}+1}^{n}(2 i+\alpha+\beta) K_{i-1}^{\left(j_{2}, j_{2}\right)}(-1,-1), \\
& \gamma_{n}=\sum_{i=\max \left\{j_{1}, j_{2}\right\}+1}^{n}(2 i+\alpha+\beta)\left[K_{i-1}^{\left(j_{1}, j_{1}\right)}(1,1) K_{i-1}^{\left(j_{2}, j_{2}\right)}(-1,-1)-\left(K_{i-1}^{\left(j_{1}, j_{2}\right)}(1,-1)\right)^{2}\right] .
\end{aligned}
$$

Now, we are ready to compute the asymptotic behavior of the above sequences of real numbers and, via (16), we obtain the wanted asymptotic behavior of the eigenvalues $\varrho_{n}$.

Proposition 2. Let $\alpha_{n}, \beta_{n}$ and $\gamma_{n}$ be defined in (18)-(20), respectively. Then,

$$
\begin{aligned}
\lim _{n \rightarrow+\infty} \frac{\alpha_{n}}{n^{2 \alpha+4 j_{1}+4}} & =\frac{1}{2^{\alpha+\beta+2 j_{1}+1}\left(\alpha+2 j_{1}+2\right)\left(\alpha+2 j_{1}+1\right) \Gamma^{2}\left(\alpha+j_{1}+1\right)}, \\
\lim _{n \rightarrow+\infty} \frac{\beta_{n}}{n^{2 \beta+4 j_{2}+4}} & =\frac{1}{2^{\alpha+\beta+2 j_{2}+1}\left(\beta+2 j_{2}+2\right)\left(\beta+2 j_{2}+1\right) \Gamma^{2}\left(\beta+j_{2}+1\right)}, \\
\lim _{n \rightarrow+\infty} \frac{\gamma_{n}}{n^{2 \alpha+2 \beta+4 j_{1}+4 j_{2}+6}} & =\mathfrak{C}_{\alpha, \beta, j_{1}, j_{2},}
\end{aligned}
$$

where $\mathfrak{C}_{\alpha, \beta, j_{1}, j_{2}}=\frac{1}{2^{2\left(\alpha+\beta+j_{1}+j_{2}+1\right)}\left(\alpha+\beta+2 j_{1}+2 j_{2}+3\right)\left(\alpha+2 j_{1}+1\right)\left(\beta+2 j_{2}+1\right) \Gamma^{2}\left(\alpha+j_{1}+1\right) \Gamma^{2}\left(\beta+j_{2}+1\right)}$.

Proof. To prove (21), we use (13a), the Stolz-Cesàro criterion and

$$
\lim _{n \rightarrow+\infty} \frac{n^{2 \alpha+4 j_{1}+4}-(n-1)^{2 \alpha+4 j_{1}+4}}{\left(2 \alpha+4 j_{1}+4\right) n^{2 \alpha+4 j_{1}+3}}=1 .
$$

Then,

$$
\begin{aligned}
\lim _{n \rightarrow+\infty} \frac{\alpha_{n}}{n^{2 \alpha+4 j_{1}+4}} & =\lim _{n \rightarrow+\infty} \frac{\alpha_{n}-\alpha_{n-1}}{n^{2 \alpha+4 j_{1}+4}-(n-1)^{2 \alpha+4 j_{1}+4}} \\
& =\lim _{n \rightarrow+\infty} \frac{(2 n+\alpha+\beta) K_{n-1}^{\left(j_{1}, j_{1}\right)}(1,1)}{\left(2 \alpha+4 j_{1}+4\right) n^{2 \alpha+4 j_{1}+3}} \\
& =\frac{1}{2^{\alpha+\beta+2 j_{1}+1}\left(\alpha+2 j_{1}+2\right)\left(\alpha+2 j_{1}+1\right) \Gamma\left(\alpha+j_{1}+1\right) \Gamma\left(\alpha+j_{1}+1\right)}
\end{aligned}
$$

Formula (22) is proved in the same way now using (13b). Finally, to establish (23), we use Proposition 1 getting

$$
\begin{aligned}
\lim _{n \rightarrow+\infty} \frac{\gamma_{n}}{n^{2 \alpha+2 \beta+4 j_{1}+4 j_{2}+6}} & =\lim _{n \rightarrow+\infty} \frac{\gamma_{n}-\gamma_{n-1}}{n^{2 \alpha+2 \beta+4 j_{1}+4 j_{2}+6}-(n-1)^{2 \alpha+2 \beta+4 j_{1}+4 j_{2}+6}} \\
& =\lim _{n \rightarrow+\infty} \frac{(2 n+\alpha+\beta)\left[K_{n-1}^{\left(j_{1}, j_{1}\right)}(1,1) K_{n-1}^{\left(j_{2}, j_{2}\right)}(-1,-1)-\left(K_{n-1}^{\left(j_{1}, j_{2}\right)}(1,-1)\right)^{2}\right]}{\left(2 \alpha+2 \beta+4 j_{1}+4 j_{2}+6\right) n^{2 \alpha+2 \beta+4 j_{1}+4 j_{2}+5}} \\
& =\mathfrak{C}_{\alpha, \beta, j_{1}, j_{2} .} .
\end{aligned}
$$

Theorem 1. Let $\varrho_{n}$ be the eigenvalues of the differential operator $\mathcal{T}$. Then,

$$
\lim _{n \rightarrow+\infty} \frac{\varrho_{n}}{n^{2 \alpha+2 \beta+4 j_{1}+4 j_{2}+6}}=M N \mathfrak{C}_{\alpha, \beta, j_{1}, j_{2}},
$$

where the quantity $\mathfrak{C}_{\alpha, \beta, j_{1}, j_{2}}$ is defined in Proposition 2. 
Proof. It is enough to apply Proposition 2 in (16).

Remark 1. Notice that, for a symmetric inner product of type (1), we cannot derive the asymptotic behavior of the corresponding eigenvalues $\varrho_{n}$ from Theorem 1 in a straightforward way. This is because the expressions for $\varrho_{n}$ are essentially different in each case (see (16) and (17)). Thus, as we have commented previously, the symmetric case is technically easier to analyze and it was done in [31].

\section{Some Properties of Discrete Jacobi-Sobolev Orthogonal Polynomials}

The aim of this section is to get useful algebraic and asymptotic properties of these discrete Sobolev orthogonal polynomials with the target of computing the asymptotic value $r_{0}$ in the next section. For convenience, now we use the orthogonal polynomials with respect to (1) with the same leading coefficient as the polynomials $P_{n}^{(\alpha, \beta)}(x)$ and we denote them by $Q_{n}^{(M, N)}(x)$. First, we get the following connection formula:

Lemma 2. We have

$$
Q_{n}^{(M, N)}(x)=P_{n}^{(\alpha, \beta)}(x)-M \Phi\left(n, j_{1}, j_{2}\right) K_{n-1}^{\left(j_{1}, 0\right)}(1, x)-N \Psi\left(n, j_{1}, j_{2}\right) K_{n-1}^{\left(j_{2}, 0\right)}(-1, x),
$$

where

$$
\begin{aligned}
& \Phi\left(n, j_{1}, j_{2}\right)=\frac{\left(P_{n}^{(\alpha, \beta)}\right)^{\left(j_{1}\right)}(1)\left(1+N K_{n-1}^{\left(j_{2}, j_{2}\right)}(-1,-1)\right)-N\left(P_{n}^{(\alpha, \beta)}\right)^{\left(j_{2}\right)}(-1) K_{n-1}^{\left(j_{1}, j_{2}\right)}(1,-1)}{1+M K_{n-1}^{\left(j_{1}, j_{1}\right)}(1,1)+N K_{n-1}^{\left(j_{2}, j_{2}\right)}(-1,-1)+M N\left(K_{n-1}^{\left(j_{1}, j_{1}\right)}(1,1) K_{n-1}^{\left(j_{2}, j_{2}\right)}(-1,-1)-\left(K_{n-1}^{\left(j_{1}, j_{2}\right)}(1,-1)\right)^{2}\right)^{2}}, \\
& \Psi\left(n, j_{1}, j_{2}\right)=\frac{\left(P_{n}^{(\alpha, \beta)}\right)^{\left(j_{2}\right)}(-1)\left(1+M K_{n-1}^{\left(j_{1}, j_{1}\right)}(1,1)\right)-M\left(P_{n}^{(\alpha, \beta)}\right)^{\left(j_{1}\right)}(1) K_{n-1}^{\left(j_{1}, j_{2}\right)}(1,-1)}{1+M K_{n-1}^{\left(j_{1}, j_{1}\right)}(1,1)+N K_{n-1}^{\left(j_{2}, j_{2}\right)}(-1,-1)+M N\left(K_{n-1}^{\left(j_{1}, j j_{1}\right)}(1,1) K_{n-1}^{\left(j_{2}, j_{2}\right)}(-1,-1)-\left(K_{n-1}^{\left(j_{1}, j_{2}\right)}(1,-1)\right)^{2}\right)} .
\end{aligned}
$$

Proof. We use a standard technique (see, for example, [37]). We have

$$
Q_{n}^{(M, N)}(x)=P_{n}^{(\alpha, \beta)}(x)+\sum_{k=0}^{n-1} a_{n, k} P_{k}^{(\alpha, \beta)}(x) .
$$

Now, using the orthogonality of $Q_{n}^{(M, N)}(x)$, we get for $0 \leq i<n$,

$$
\begin{aligned}
0 & =\left(Q_{n}^{(M, N)}(x), P_{i}^{(\alpha, \beta)}(x)\right)_{S}=\left(P_{n}^{(\alpha, \beta)}(x)+\sum_{k=0}^{n-1} a_{n, k} P_{k}^{(\alpha, \beta)}(x), P_{i}^{(\alpha, \beta)}(x)\right)_{S} \\
& =\int_{-1}^{1} P_{n}^{(\alpha, \beta)}(x) P_{i}^{(\alpha, \beta)}(x)(1-x)^{\alpha}(1+x)^{\beta} d x \\
& +\sum_{k=0}^{n-1} a_{n, k} \int_{-1}^{1} P_{k}^{(\alpha, \beta)}(x) P_{i}^{(\alpha, \beta)}(x)(1-x)^{\alpha}(1+x)^{\beta} d x \\
& +M\left[\left(Q_{n}^{(M, N)}\right)^{\left(j_{1}\right)}(1)\left(P_{i}^{(\alpha, \beta)}\right)^{\left(j_{1}\right)}(1)\right]+N\left[\left(Q_{n}^{(M, N)}\right)^{\left(j_{2}\right)}(-1)\left(P_{i}^{(\alpha, \beta)}\right)^{\left(j_{2}\right)}(-1)\right] \\
& =a_{n, i}\left\|P_{i}^{(\alpha, \beta)}\right\|_{\alpha}^{2}+M\left(Q_{n}^{(M, N)}\right)^{\left(j_{1}\right)}(1)\left(P_{i}^{(\alpha, \beta)}\right)^{\left(j_{1}\right)}(1)+N\left(Q_{n}^{(M, N)}\right)^{\left(j_{2}\right)}(-1)\left(P_{i}^{(\alpha, \beta)}\right)^{\left(j_{2}\right)}(-1) .
\end{aligned}
$$

Then,

$$
a_{n, i}=-\frac{M\left(Q_{n}^{(M, N)}\right)^{\left(j_{1}\right)}(1)\left(P_{i}^{(\alpha, \beta)}\right)^{\left(j_{1}\right)}(1)+N\left(Q_{n}^{(M, N)}\right)^{\left(j_{2}\right)}(-1)\left(P_{i}^{(\alpha, \beta)}\right)^{\left(j_{2}\right)}(-1)}{\left\|P_{i}^{(\alpha, \beta)}\right\|^{2}} .
$$


Next, using the definition of kernel polynomials and the expression (27), we obtain:

$$
\begin{aligned}
Q_{n}^{(M, N)}(x)= & P_{n}^{(\alpha, \beta)}(x)-M\left(Q_{n}^{(M, N)}\right)^{\left(j_{1}\right)}(1) K_{n-1}^{\left(j_{1}, 0\right)}(1, x) \\
& -N\left(Q_{n}^{(M, N)}\right)^{\left(j_{2}\right)}(-1) K_{n-1}^{\left(j_{2}, 0\right)}(-1, x) .
\end{aligned}
$$

We need to calculate $\left(Q_{n}^{(M, N)}\right)^{\left(j_{1}\right)}(1)$ and $\left(Q_{n}^{(M, N)}\right)^{\left(j_{2}\right)}(-1)$. To do this, we build a system of two equations and two unknowns as it follows. We derive the expression (28) $j_{1}$ times and evaluate at $x=1$, then we repeat the process, but now deriving it $j_{2}$ times and evaluating at $x=-1$. Thus, using the fact that

$$
K_{n-1}^{(j, k)}(a, b)=K_{n-1}^{(k, j)}(b, a),
$$

for all $a, b \in \mathbb{R}$ and $j, k \in \mathbb{N} \cup\{0\}$, we can build the linear system

$$
\begin{aligned}
\left(Q_{n}^{(M, N)}\right)^{\left(j_{1}\right)}(1)= & \left(P_{n}^{(\alpha, \beta)}\right)^{\left(j_{1}\right)}(1)-M\left(Q_{n}^{(M, N)}\right)^{\left(j_{1}\right)}(1) K_{n-1}^{\left(j_{1}, j_{1}\right)}(1,1) \\
& -N\left(Q_{n}^{(M, N)}\right)^{\left(j_{2}\right)}(-1) K_{n-1}^{\left(j_{2}, j_{1}\right)}(-1,1), \\
\left(Q_{n}^{(M, N)}\right)^{\left(j_{2}\right)}(-1)= & \left(P_{n}^{(\alpha, \beta)}\right)^{\left(j_{2}\right)}(-1)-M\left(Q_{n}^{(M, N)}\right)^{\left(j_{1}\right)}(1) K_{n-1}^{\left(j_{2}, j_{1}\right)}(-1,1) \\
& -N\left(Q_{n}^{(M, N)}\right)^{\left(j_{2}\right)}(-1) K_{n-1}^{\left(j_{2}, j_{2}\right)}(-1,-1) .
\end{aligned}
$$

Solving the system, we get the result.

The asymptotic behavior of the coefficients in the connection Formula (24) is given by the following result.

Lemma 3. Let $\Phi\left(n, j_{1}, j_{2}\right)$ and $\Psi\left(n, j_{1}, j_{2}\right)$ be defined in (25) and (26), respectively. Then,

$$
\begin{aligned}
\lim _{n \rightarrow+\infty} \Phi\left(n, j_{1}, j_{2}\right) n^{\alpha+2 j_{1}+2} & =\frac{2^{\alpha+\beta+j_{1}+1}\left(\alpha+2 j_{1}+1\right) \Gamma\left(\alpha+j_{1}+1\right)}{M}, \\
\lim _{n \rightarrow+\infty}(-1)^{n} \Psi\left(n, j_{1}, j_{2}\right) n^{\beta+2 j_{2}+2} & =\frac{(-1)^{j_{2}} 2^{\alpha+\beta+j_{2}+1}\left(\beta+2 j_{2}+1\right) \Gamma\left(\beta+j_{2}+1\right)}{N} .
\end{aligned}
$$


Proof. To establish the results, we need (10), (11), and (13). We have only proved (29) because the proof of (30) is totally similar. Thus,

$$
\begin{aligned}
& \lim _{n \rightarrow+\infty} \Phi\left(n, j_{1}, j_{2}\right) n^{\alpha+2 j_{1}+2} \\
= & \frac{\left(P_{n}^{(\alpha, \beta)}\right)^{\left(j_{1}\right)}(1) n^{\alpha+2 j_{1}+2}}{1+M K_{n-1}^{\left(j_{1}, j_{1}\right)}(1,1)+N K_{n-1}^{\left(j_{2}, j_{2}\right)}(-1,-1)+M N\left(K_{n-1}^{\left(j_{1}, j_{1}\right)}(1,1) K_{n-1}^{\left(j_{2}, j_{2}\right)}(-1,-1)-\left(K_{n-1}^{\left(j_{1}, j_{2}\right)}(1,-1)\right)^{2}\right)} \\
+ & \frac{N\left(P_{n}^{(\alpha, \beta)}\right)^{\left(j_{1}\right)}(1) K_{n-1}^{\left(j_{2}, j_{2}\right)}(-1,-1) n^{\alpha+2 j_{1}+2}}{1+M K_{n-1}^{\left(j_{1}, j_{1}\right)}(1,1)+N K_{n-1}^{\left(j_{2}, j_{2}\right)}(-1,-1)+M N\left(K_{n-1}^{\left(j_{1}, j_{1}\right)}(1,1) K_{n-1}^{\left(j_{2}, j_{2}\right)}(-1,-1)-\left(K_{n-1}^{\left(j_{1}, j_{2}\right)}(1,-1)\right)^{2}\right)} \\
- & \frac{N\left(P_{n}^{(\alpha, \beta)}\right)^{\left(j_{2}\right)}(-1) K_{n-1}^{\left(j_{1}, j_{2}\right)}(1,-1) n^{\alpha+2 j_{1}+2}}{1+M K_{n-1}^{\left(j_{1}, j_{1}\right)}(1,1)+N K_{n-1}^{\left(j_{2}, j_{2}\right)}(-1,-1)+M N\left(K_{n-1}^{\left(j_{1}, j_{1}\right)}(1,1) K_{n-1}^{\left(j_{2}, j_{2}\right)}(-1,-1)-\left(K_{n-1}^{\left(j_{1}, j_{2}\right)}(1,-1)\right)^{2}\right)} \\
= & \lim _{n \rightarrow+\infty} \frac{N\left(P_{n}^{(\alpha, \beta)}\right)}{\left(j_{1}\right)}(1) K_{n-1}^{\left(j_{2}, j_{2}\right)}(-1,-1) \frac{n^{\alpha+2 j_{1}+2}}{n^{2 \alpha+2 \beta+4 j_{1}+4 j_{2}+4}} \\
= & \frac{2^{\alpha+\beta+j_{1}+1}\left(\alpha+2 K_{n-1}^{\left(j_{1}, j_{1}\right)}(1,1) K_{n-1}^{\left(j_{2}, j_{2}\right)}(-1,-1)\right.}{n^{2 \alpha+2 \beta+4 j_{1}+4 j_{2}+4}} \\
M & \Gamma\left(\alpha+j_{1}+1\right)
\end{aligned}
$$

The following result compares asymptotically $\left(Q_{n}^{(M, N)}\right)^{(k)}(x)$ and $\left(P_{n}^{(\alpha, \beta)}\right)^{(k)}(x)$ at the end points of the interval $[-1,1]$.

Lemma 4. Let $k$ be a nonnegative integer; then,

$$
\begin{aligned}
& \lim _{n \rightarrow+\infty} \frac{\left(Q_{n}^{(M, N)}\right)^{(k)}(1)}{\left(P_{n}^{(\alpha, \beta)}\right)^{(k)}(1)}=\frac{k-j_{1}}{\alpha+j_{1}+k+1}, \\
& \lim _{n \rightarrow+\infty} \frac{\left(Q_{n}^{(M, N)}\right)^{(k)}(-1)}{\left(P_{n}^{(\alpha, \beta)}\right)^{(k)}(-1)}=\frac{k-j_{2}}{\beta+j_{2}+k+1} .
\end{aligned}
$$


Proof. We only prove (31) because (32) is obtained in the same way. We derive the Formula (24) $k$ times and evaluate at $x=1$, then we take limits and apply Propositon 1 and Lemmas 1 and 3 getting

$$
\begin{aligned}
& \lim _{n \rightarrow+\infty} \frac{\left(Q_{n}^{(M, N)}\right)^{(k)}(1)}{\left(P_{n}^{(\alpha, \beta)}\right)^{(k)}(1)} \\
= & \lim _{n \rightarrow+\infty} \frac{\left(P_{n}^{(\alpha, \beta)}\right)^{(k)}(1)-M \Phi\left(n, j_{1}, j_{2}\right) K_{n-1}^{\left(j_{1}, k\right)}(1,1)-N \Psi\left(n, j_{1}, j_{2}\right) K_{n-1}^{\left(j_{2}, k\right)}(-1,1)}{\left(P_{n}^{(\alpha, \beta)}\right)^{(k)}(1)} \\
= & 1-M \frac{\frac{2^{\alpha+\beta+j_{1}+1}\left(\alpha+2 j_{1}+1\right) \Gamma\left(\alpha+j_{1}+1\right)}{M} \frac{1}{2^{\alpha+\beta+j_{1}+k+1}\left(\alpha+j_{1}+k+1\right) \Gamma\left(\alpha+j_{1}+1\right) \Gamma(\alpha+k+1)}}{\frac{1}{2^{k} \Gamma(\alpha+k+1)}} \\
= & 1-\frac{\alpha+2 j_{1}+1}{\alpha+j_{1}+k+1}=\frac{k-j_{1}}{\alpha+j_{1}+k+1} .
\end{aligned}
$$

Remark 2. From Lemmas 1 and 4, we deduce that

$$
\begin{gathered}
Q_{n}^{(M, N)}(1) \approx \frac{-j_{1}}{\alpha+j_{1}+1} P_{n}^{(\alpha, \beta)}(1) \approx \frac{-j_{1}}{\left(\alpha+j_{1}+1\right) \Gamma(\alpha+1)} n^{\alpha}, \quad \text { if } j_{1}>0, \\
Q_{n}^{(M, N)}(-1) \approx \frac{-j_{2}}{\beta+j_{2}+1} P_{n}^{(\alpha, \beta)}(-1) \approx \frac{(-1)^{n+1} j_{2}}{\left(\beta+j_{2}+1\right) \Gamma(\beta+1)} n^{\beta}, \quad \text { if } j_{2}>0,
\end{gathered}
$$

where the symbol $\approx$ means the following: if $a_{n} \approx b_{n}$, then $\lim _{n \rightarrow+\infty} \frac{a_{n}}{b_{n}}=1$.

We claim that the Euclidean norm of the Sobolev polynomials behaves asymptotically like the one of classical Jacobi polynomials.

\section{Proposition 3.}

$$
\lim _{n \rightarrow+\infty} \frac{\left\|Q_{n}^{(M, N)}\right\|_{S}}{\left\|P_{n}^{(\alpha, \beta)}\right\|}=1 .
$$

Proof. We have

$$
\begin{aligned}
\left(Q_{n}^{(M, N)}(x), Q_{n}^{(M, N)}(x)\right)_{S} & =\left(Q_{n}^{(M, N)}(x), P_{n}^{(\alpha, \beta)}(x)\right)_{S} \\
& =\left\|P_{n}^{(\alpha, \beta)}\right\|^{2}+M\left(Q_{n}^{(M, N)}\right)^{\left(j_{1}\right)}(1)\left(P_{n}^{(\alpha, \beta)}\right)^{\left(j_{1}\right)} \\
& +N\left(Q_{n}^{(M, N)}\right)^{\left(j_{2}\right)}(-1)\left(P_{n}^{(\alpha, \beta)}\right)^{\left(j_{2}\right)}(-1) .
\end{aligned}
$$

Then,

$$
\begin{aligned}
\frac{\left\|Q_{n}^{(M, N)}\right\|_{S}^{2}}{\left\|P_{n}^{(\alpha, \beta)}\right\|^{2}} & =1+M \frac{\left(Q_{n}^{(M, N)}\right)^{\left(j_{1}\right)}(1)\left(P_{n}^{(\alpha, \beta)}\right)^{\left(j_{1}\right)}(1)}{\left\|P_{n}^{(\alpha)}\right\|^{2}}+N \frac{\left(Q_{n}^{(M, N)}\right)^{\left(j_{2}\right)}(-1)\left(P_{n}^{(\alpha, \beta)}\right)^{\left(j_{2}\right)}(-1)}{\left\|P_{n}^{(\alpha)}\right\|^{2}} \\
& =1+M \frac{\Phi\left(n, j_{1}, j_{2}\right)\left(P_{n}^{(\alpha, \beta)}\right)^{\left(j_{1}\right)}(1)}{\left\|P_{n}^{(\alpha)}\right\|^{2}}+N \frac{\Psi\left(n, j_{1}, j_{2}\right)\left(P_{n}^{(\alpha, \beta)}\right)^{\left(j_{2}\right)}(-1)}{\left\|P_{n}^{(\alpha)}\right\|^{2}} .
\end{aligned}
$$


Finally, taking limits and using the relations (10)-(12), (29) and (30), we get

$$
\lim _{n \rightarrow+\infty} \frac{\Phi\left(n, j_{1}, j_{2}\right)\left(P_{n}^{(\alpha, \beta)}\right)^{\left(j_{1}\right)}(1)}{\left\|P_{n}^{(\alpha)}\right\|^{2}}=\lim _{n \rightarrow+\infty} \frac{\Psi\left(n, j_{1}, j_{2}\right)\left(P_{n}^{(\alpha, \beta)}\right)^{\left(j_{2}\right)}(-1)}{\left\|P_{n}^{(\alpha)}\right\|^{2}}=0,
$$

and the result is proved.

A useful estimate of the uniform norm is given in the next result.

Theorem 2. Let $q=\max \{\alpha, \beta\}$ be. If $q \geq-1 / 2$, then we have

$$
\left\|Q_{n}^{(M, N)}\right\|_{\infty}=\max _{x \in[-1,1]}\left|Q_{n}^{(M, N)}(x)\right| \leq \mathcal{C} n^{q},
$$

where $\mathcal{C}$ is a constant independent of $n$.

Proof. We have forwardly from (24)

$$
\begin{aligned}
\max _{x \in[-1,1]}\left|Q_{n}^{(M, N)}(x)\right| & \leq \max _{x \in[-1,1]}\left|P_{n}^{(\alpha, \beta)}(x)\right|+M\left|\Phi\left(n, j_{1}, j_{2}\right)\right| \max _{x \in[-1,1]}\left|K_{n-1}^{\left(j_{1}, 0\right)}(1, x)\right| \\
& +N\left|\Psi\left(n, j_{1}, j_{2}\right)\right| \max _{x \in[-1,1]}\left|K_{n-1}^{\left(j_{2}, 0\right)}(-1, x)\right| .
\end{aligned}
$$

First, let us find asymptotic upper bounds of the quantities $\max _{x \in[-1,1]}\left|K_{n-1}^{\left(j_{1}, 0\right)}(1, x)\right|$ and $\max _{x \in[-1,1]}\left|K_{n-1}^{\left(j_{2}, 0\right)}(-1, x)\right|$, to find an upper bound of $\max _{x \in[-1,1]}\left|Q_{n}^{(M, N)}(x)\right|$. To reach this, we use the well-known upper bound for the classical Jacobi polynomials (see ([32], f. (7.32.2))):

$$
\max _{x \in[-1,1]}\left|P_{n}^{(\alpha, \beta)}(x)\right|=\left(\begin{array}{c}
n+q \\
n
\end{array}\right) \approx \frac{n^{q}}{\Gamma(q+1)}, \quad \text { if } \quad q \geq-1 / 2 .
$$

Thus,

$$
\begin{aligned}
\max _{x \in[-1,1]}\left|K_{n-1}^{\left(j_{1}, 0\right)}(1, x)\right| & =\max _{x \in[-1,1]}\left|\sum_{i=0}^{n-1} \frac{\left(P_{i}^{(\alpha, \beta)}\right)^{\left(j_{1}\right)}(1) P_{i}^{(\alpha, \beta)}(x)}{\left\|P_{i}^{(\alpha, \beta)}(x)\right\|^{2}}\right| \\
& \leq \sum_{i=0}^{n-1} \frac{\left(P_{i}^{(\alpha, \beta)}\right)^{\left(j_{1}\right)}(1) \max _{x \in[-1,1]}\left|P_{i}^{(\alpha, \beta)}(x)\right|}{\left\|P_{i}^{(\alpha, \beta)}(x)\right\|^{2}} \\
& =\sum_{i=0}^{n-1} \frac{\left(\begin{array}{c}
i+q \\
i
\end{array}\right)\left(P_{i}^{(\alpha, \beta)}\right)^{\left(j_{1}\right)}(1)}{\left\|P_{i}^{(\alpha, \beta)}(x)\right\|^{2}} \\
& =\frac{1}{\Gamma(q+1)} \sum_{i=0}^{n-1} \frac{\Gamma(i+q+1)\left(P_{i}^{(\alpha, \beta)}\right)\left(j_{1}\right)}{\Gamma(i+1)\left\|P_{i}^{(\alpha, \beta)}(x)\right\|^{2}} \\
& \approx \frac{n^{\alpha+2 j_{1}+q+2}}{2^{\alpha+\beta+j_{1}}\left(\alpha+2 j_{1}+q+2\right) \Gamma\left(\alpha+j_{1}+1\right) \Gamma(q+1)}
\end{aligned}
$$


where we have used the Stolz-Cesàro criterion, and formulas (9), (10), and (12) to prove

$$
\begin{aligned}
\lim _{n \rightarrow+\infty} \frac{\sum_{i=0}^{n-1} \frac{\Gamma(i+q+1)\left(P_{i}^{(\alpha, \beta)}\right)^{\left(j_{1}\right)}(1)}{\Gamma(i+1)\left\|P_{i}^{(\alpha, \beta)}(x)\right\|^{2}}}{n^{\alpha+q+2 j_{1}+2}} & =\lim _{n \rightarrow+\infty} \frac{\left(P_{n-1}^{(\alpha, \beta)}\right)^{\left(j_{1}\right)}(1) \Gamma(n+q)}{\left(\alpha+q+2 j_{1}+2\right) \Gamma(n)\left\|P_{n-1}^{(\alpha, \beta)}(x)\right\|^{2} n^{\alpha+q+2 j_{1}+1}} \\
& =\frac{1}{2^{\alpha+\beta+j_{1}\left(\alpha+q+2 j_{1}+2\right) \Gamma\left(\alpha+j_{1}+1\right)} .}
\end{aligned}
$$

In an analogous way,

$$
\begin{aligned}
\max _{x \in[-1,1]}\left|K_{n-1}^{\left(j_{2}, 0\right)}(-1, x)\right| & \leq \frac{1}{\Gamma(q+1)} \sum_{i=0}^{n-1} \frac{\Gamma(i+q+1)\left|\left(P_{i}^{(\alpha, \beta)}\right)^{\left(j_{2}\right)}(-1)\right|}{\Gamma(i+1)\left\|P_{i}^{(\alpha, \beta)}(x)\right\|^{2}} \\
& \approx \frac{n^{\beta+2 j_{2}+q+2}}{2^{\alpha+\beta+j_{2}}\left(\beta+2 j_{2}+q+2\right) \Gamma\left(\beta+j_{2}+1\right) \Gamma(q+1)},
\end{aligned}
$$

where now we have used the Stolz-Cesàro criterion again, and formulas (9), (11) and (12) to prove

$$
\begin{aligned}
\lim _{n \rightarrow+\infty} \frac{\sum_{i=0}^{n-1} \frac{\Gamma(i+q+1)\left|\left(P_{i}^{(\alpha, \beta)}\right)^{\left(j_{2}\right)}(-1)\right|}{\Gamma(i+1)\left\|P_{i}^{(\alpha, \beta)}(x)\right\|^{2}}}{n^{\beta+q+2 j_{2}+2}} & =\lim _{n \rightarrow+\infty} \frac{\Gamma(n+q)\left|\left(P_{n-1}^{(\alpha, \beta)}\right)^{\left(j_{2}\right)}(-1)\right|}{\left.\left(\beta+q+2 j_{2}+2\right) \Gamma(n)\left\|P_{n-1}^{(\alpha, \beta)}(x)\right\|\right|^{2} n^{\beta+q+2 j_{2}+1}} \\
& =\frac{1}{2^{\alpha+\beta+j_{2}\left(\beta+q+2 j_{2}+2\right) \Gamma\left(\beta+j_{2}+1\right)}} .
\end{aligned}
$$

Finally, it is enough to apply these results together with (29) and (30) in (33) to deduce the result.

Remark 3. For the case $q<-1 / 2$, we have not obtained any result. When the inner product (1) is symmetric, we can obtain an upper bound for this uniform norm (see [31]). The key in that case is that the Sobolev polynomials can be expressed in terms of a finite combination of classical Gegenbauer polynomials where the coefficients of this expansion are convergent when $n$ tends to $\infty$. Unfortunately, we have not been able to get such type of expansion for the nonsymmetric case that we are tackling in this paper. Thus, this case remains open.

\section{Asymptotics of the Value $r_{0}$}

As we have commented in the introduction, in our case, if we compute the value of $s_{0}$, then we can guarantee the existence of $r_{0}$ and obtain its explicit value.

Theorem 3. Let $q=\max \{\alpha, \beta\} \geq-1 / 2$ be. If $q=\alpha$ and $j_{1}>0$ or $q=\beta$ and $j_{2}>0$, then

$$
s_{0}:=\lim _{n \rightarrow+\infty} \frac{\log \left(\max _{x \in[-1,1]}\left|q_{n}^{(M, N)}(x)\right|\right)}{\log \left(\varrho_{n}\right)}=\frac{q+1 / 2}{2\left(\alpha+\beta+2 j_{1}+2 j_{2}+3\right)}<1,
$$

where $q_{n}^{(M, N)}(x)$ denotes the orthonormal polynomials with respect to (1).

Proof. Taking into account Theorem 2, Remark 2 and the hypothesis of this theorem, we can assure readers that there are two positive constants $C_{1}$ and $C_{2}$ independent of $n$, so that

$$
C_{1} n^{q} \leq \max _{x \in[-1,1]}\left|Q_{n}^{(M, N)}(x)\right| \leq C_{2} n^{q}
$$


In this way, simple algebraic manipulations lead to

$$
\begin{aligned}
& \frac{\log \left(\max _{x \in[-1,1]}\left|q_{n}^{(M, N)}(x)\right|\right)}{\log \left(\varrho_{n}\right)}=\frac{\log \left(\max _{x \in[-1,1]}\left|Q_{n}^{(M, N)}(x)\right|\right)-\log \left(\left\|Q_{n}^{(M, N)}\right\|_{S}\right)}{\log \left(\varrho_{n}\right)} \\
= & \frac{\log \left(\frac{\max _{x \in[-1,1]}\left|Q_{n}^{(M, N)}(x)\right|}{n^{q}}\right)+\log \left(n^{q}\right)-\left(\log \left(\left\|Q_{n}^{(M, N)}\right\|_{S} n^{1 / 2}\right)-\log \left(n^{1 / 2}\right)\right)}{\log \left(\frac{Q_{n}}{n^{2\left(\alpha+\beta+2 j_{1}+2 j_{2}+3\right)}}\right)+\log \left(n^{2\left(\alpha+\beta+2 j_{1}+2 j_{2}+3\right)}\right)} \\
= & \frac{\log \left(\frac{\max _{x \in[-1,1]}\left|Q_{n}^{(M, N)}(x)\right|}{n^{q}}\right)+q \log (n)-\left(\log \left(\left\|Q_{n}^{(M, N)}\right\|_{S} n^{1 / 2}\right)-1 / 2 \log (n)\right)}{\log \left(\frac{\varrho_{n}}{n^{2\left(\alpha+\beta+2 j_{1}+2 j_{2}+3\right)}}\right)+2\left(\alpha+\beta+2 j_{1}+2 j_{2}+3\right) \log (n)} .
\end{aligned}
$$

Now, applying (34), the first term in the numerator of the above fraction is bounded, so taking limits we get the result.

Corollary 1. Let $q=\max \{\alpha, \beta\} \geq-1 / 2$ be. If $q=\alpha$ and $j_{1}>0$ or $q=\beta$ and $j_{2}>0$, then

$$
r_{0}=\lim _{n \rightarrow+\infty} \frac{\log \left(n \max _{x \in[-1,1]}\left|q_{n}^{(M, N)}(x)\right|^{2}\right)}{\log \left(\varrho_{n}\right)}=2 s_{0}+\frac{1}{2\left(\alpha+\beta+2 j_{1}+2 j_{2}+3\right)}=\frac{2(q+1)}{2\left(\alpha+\beta+2 j_{1}+2 j_{2}+3\right)} .
$$

Proof. We can observe

$$
\begin{aligned}
& \frac{\log \left(n \max _{x \in[-1,1]}\left|q_{n}^{(M, N)}(x)\right|^{2}\right)}{\log \left(\varrho_{n}\right)} \\
= & \frac{\log (n)}{\log \left(\frac{\varrho_{n}}{n^{2\left(\alpha+\beta+2 j_{1}+2 j_{2}+3\right)}}\right)+2\left(\alpha+\beta+2 j_{1}+2 j_{2}+3\right) \log (n)} \\
+ & \frac{\log \left(\max _{x \in[-1,1]}\left|q_{n}^{(M, N)}(x)\right|^{2}\right)}{\log \left(\frac{\varrho_{n}}{n^{2\left(\alpha+\beta+2 j_{1}+2 j_{2}+3\right)}}\right)+2\left(\alpha+\beta+2 j_{1}+2 j_{2}+3\right) \log (n)} .
\end{aligned}
$$

We claim that the limits when $n$ tends to infinite of the two terms in the previous expression exist, and therefore we have proven the first equality in (35). Using Theorem 1, the limit of the first term exists and its value is

$$
\lim _{n \rightarrow+\infty} \frac{\log (n)}{\log \left(\frac{Q_{n}}{n^{2\left(\alpha+\beta+2 j_{1}+2 j_{2}+3\right)}}\right)+2\left(\alpha+\beta+2 j_{1}+2 j_{2}+3\right) \log (n)}=\frac{1}{2\left(\alpha+\beta+2 j_{1}+2 j_{2}+3\right)} .
$$

To establish the existence of the other limit, it is enough to proceed in the same way as the proof of Theorem 3 but taking into account that now we have $\max _{x \in[-1,1]}\left|q_{n}^{(M, N)}(x)\right|^{2}$. The square modifies the previous proof slightly. Anyway, we can prove that the limit exists and we get

$$
\lim _{n \rightarrow+\infty} \frac{\log \left(\max _{x \in[-1,1]}\left|q_{n}^{(M, N)}(x)\right|^{2}\right)}{\log \left(\frac{Q_{n}}{n^{2\left(\alpha+\beta+2 j_{1}+2 j_{2}+3\right)}}\right)+2\left(\alpha+\beta+2 j_{1}+2 j_{2}+3\right) \log (n)}=2 s_{0} .
$$

Gathering (36) and (37), we obtain the result.

Remark 4. Notice that, under the hypothesis of Theorem $3, s_{0} \in[0,1)$, but $r_{0}$ is strictly positive, i.e., $r_{0} \in(0,1)$. 
Remark 5. To establish Theorem 3, it is essential to have an upper and lower bound of the uniform norm of $Q_{n}^{(M, N)}$. Moreover, these bounds must be of the same order. That occurs when $j_{1}$ or $j_{2}$ are positive integers although when both $j_{1}$ and $j_{2}$ are zero (Krall case), we cannot guarantee it. In this case, all the zeros of the Sobolev polynomials are within the interval $(-1,1)$ and both limits in Lemma 4 are zero when $k=0$. However, to establish the bounds that permit proving Theorem 3, it is necessary that these limits do not vanish. We have not found the adequate techniques to tackle this problem, so the existence of $r_{0}$ in the Krall case is posed as an open question.

Remark 6. As we have commented in Remark 1, in the symmetric case, the limit value $r_{0}$ was obtained in [31].

Remark 7. We have made numerical experiments with the objective of computing experimentally the values $r_{0}$ and $s_{0}$. All the computations have been done with the software Mathematica ${ }^{\circledR} 12$ (Wolfram Research Europe Ltd, Long Hanborough Oxfordshire, United Kingdom). We have checked the convergence, but we have observed that it is slow, for example, taking $\alpha=3, \beta=1 / 2, j_{1}=1, j_{2}=2, M=4$, and $N=5$, we get

\begin{tabular}{cll}
\hline & $\frac{\log \left(\max _{x \in[-1,1]}\left|q_{n}^{(M, N)}(x)\right|\right)}{\log \left(\varrho_{n}\right)}$ & $\frac{\log \left(n \max _{x \in[-1,1]}\left|q_{n}^{(M, N)}(x)\right|^{2}\right)}{\log \left(\varrho_{n}\right)}$ \\
\cline { 2 - 3 }$n=100$ & 0.125076 & 0.29941 \\
$n=400$ & 0.128414 & 0.303729 \\
$n=1600$ & 0.130748 & 0.306954 \\
Limit & $s_{0}=7 / 50=0.14$ & $r_{0}=8 / 25=0.32$ \\
\hline
\end{tabular}

Another example taking $\alpha=2, \beta=-1 / 2, j_{1}=3, j_{2}=1, M=2$, and $N=5$, we obtain

\begin{tabular}{cll}
\hline & $\frac{\log \left(\max _{x \in[-1,1]}\left|q_{n}^{(M, N)}(x)\right|\right)}{\log \left(\varrho_{n}\right)}$ & $\frac{\log \left(n \max _{x \in[-1,1]}\left|q_{n}^{(M, N)}(x)\right|^{2}\right)}{\log \left(\varrho_{n}\right)}$ \\
\hline$n=100$ & 0.104015 & 0.257152 \\
$n=400$ & 0.102267 & 0.251277 \\
$n=1600$ & 0.101653 & 0.248629 \\
Limit & $s_{0}=1 / 10=0.10$ & $r_{0}=6 / 25=0.24$ \\
\hline
\end{tabular}

Finally, we have checked numerically that, when the hypothesis of Theorem 3 is not satisfied, then the thesis of the theorem will not necessarily be fullfilled. For example, with $\alpha=3, \beta=1 / 2, j_{1}=0, j_{2}=1, M=4$, and $N=7$, we have the following results:

\begin{tabular}{cll}
\hline & $\frac{\log \left(\max _{x \in[-1,1]}\left|q_{n}^{(M, N)}(x)\right|\right)}{\log \left(\varrho_{n}\right)}$ & $\frac{\log \left(n \max _{x \in[-1,1]}\left|q_{n}^{(M, N)}(x)\right|^{2}\right)}{\log \left(\varrho_{n}\right)}$ \\
\cline { 2 - 3 }$n$ & & 0.299861 \\
$n=100$ & 0.114743 & 0.209214 \\
$n=400$ & 0.0708432 & 0.164716 \\
$n=1600$ & 0.0494866 & $r_{0}=8 / 17 \approx 0.470588235$ \\
\hline Limit & $s_{0}=7 / 34 \approx 0.20588235$ & \\
\hline
\end{tabular}

\section{Conclusions}

We have considered the nonsymmetric discrete SIP

$$
(f, g)_{S}=\int_{-1}^{1} f(x) g(x)(1-x)^{\alpha}(1+x)^{\beta} d x+M f^{\left(j_{1}\right)}(1) g^{\left(j_{1}\right)}(1)+N f^{\left(j_{2}\right)}(-1) g^{\left(j_{2}\right)}(-1)
$$

where $M, N>0, \alpha, \beta>-1$, and $j_{1}, j_{2} \in \mathbb{N} \cup\{0\}$, which can be seen as a differential perturbation located at the end points of the support of the Jacobi measure of the standard inner product 


$$
(f, g)=\int_{-1}^{1} f(x) g(x)(1-x)^{\alpha}(1+x)^{\beta} d x .
$$

It is known that these Sobolev polynomials are eigenfunctions of a differential operator. We have established the asymptotic behavior of the corresponding eigenvalues in Theorem 1, and we claim that the growth of these eigenvalues is faster than the one of the eigenvalues related to the Jacobi polynomials, concretely

\begin{tabular}{ccc}
\hline & Classical Jacobi & Discrete Jacobi-Sobolev \\
\hline Asymptotic behavior of the eigenvalues & $n^{2}$ & $n^{2 \alpha+2 \beta+4 j_{1}+4 j_{2}+6}$ \\
\hline
\end{tabular}

The study of the Jacobi-Sobolev orthogonal polynomials allows us to prove in Theorem 2 an upper bound for the uniform norm of these polynomials when $\max \{\alpha, \beta\} \geq-1 / 2$. This fact together with Theorem 1 are the essential keys to establish in Theorem 3, under some hypothesis, the value of the limit

$$
\lim _{n \rightarrow+\infty} \frac{\log \left(\max _{x \in[-1,1]}\left|q_{n}^{(M, N)}(x)\right|\right)}{\log \left(\varrho_{n}\right)}
$$

where $q_{n}^{(M, N)}(x)$ are the discrete Jacobi-Sobolev orthonormal polynomials and $\varrho_{n}$ the corresponding eigenvalues. Furthermore, three open problems are posed:

- Theorem 3 holds for $\max \{\alpha, \beta\} \geq-1 / 2$, and the case when $\max \{\alpha, \beta\} \in(-1,-1 / 2)$ remains open.

- Theorem 3 has not been established for the Krall case, that is, when $j_{1}=j_{2}=0$. Therefore, it is another open problem.

- Numerical experiments lead us to think that Theorem 3 does not hold if we relax the hypothesis. Thus, the question is: what is the value of the limit, if it exists, when either $\max \{\alpha, \beta\}=\alpha$ and $j_{1}=0$ or $\max \{\alpha, \beta\}=\beta$ and $j_{2}=0$ ?

Finally, we recall that the symmetric case was tackled in [31].

Author Contributions: All authors contributed equally to this work, as well as to its preparation. All authors have read and agreed to the published version of the manuscript.

Funding: The authors J.F.M-M. and J.J.M-B. are partially supported by the Ministry of Science, Innovation, and Universities of Spain and the European Regional Development Fund (ERDF), grant MTM2017-89941-P; they are also partially supported by ERDF and Consejería de Economía, Conocimiento, Empresas y Universidad de la Junta de Andalucía (grant UAL18-FQM-B025-A) and by Research Group FQM-0229 (belonging to Campus of International Excellence CEIMAR). The author J.J.M-B. is also partially supported by the research centre CDTIME of Universidad de Almería and by Junta de Andalucía and ERDF, Ref. SOMM17/6105/UGR.

Acknowledgments: We thank Lance Littlejohn for his help and for introducing us to this topic.

Conflicts of Interest: The authors declare no conflict of interest.

\section{References}

1. Lewis, D.C. Polynomial least square approximations. Am. J. Math. 1947, 69, 273-278. [CrossRef]

2. Schäfke, F.W.; Wolf, G. Einfache verallgemeinerte klassische Orthogonalpolynome. J. Reine Angew. Math. 1973, 262, 339-355.

3. Althammer, P. Eine Erweiterung des Orthogonalitätsbegriffes bei Polynomen und deren Anwendung auf die beste Approximation. J. Reine Angew. Math. 1962, 211, 192-204.

4. Gröbner, W. Orthogonale Polynomsysteme, die Gleichzeitig mit $f(x)$ auch deren Ableitung $f^{\prime}(x)$ approximieren. In Funktionalanalysis, Approximationstheorie, Numerische Mathematik; Collatz, L., Ed.; ISNM 7; Birkhäuser: Basel, Switzerland, 1967; pp. 24-32.

5. Marcellán, F.; Xu, Y. On Sobolev orthogonal polynomials. Expos. Math. 2015, 33, 308-352. [CrossRef] 
6. Iserles, A.; Koch, P.E.; Nørsett, S.P.; Sanz-Serna, J.M. On polynomials orthogonal with respect to certain Sobolev inner products. J. Approx. Theory 1991, 65, 151-175. [CrossRef]

7. Iserles, A; Sanz-Serna, J.M.; Koch, P.E.; Nørsett, S.P. Orthogonality and approximation in a Sobolev space. In Algorithms for Approximation, II (Shrivenham, 1988); Chapman and Hall: London, UK, 1990; pp. 117-124.

8. Marcellán, F.; Moreno-Balcázar, J.J.; Martínez-Finkelshtein, A. $k$-coherence of measures with non-classical weights. In Margarita Mathematica en Memoria de José Javier Guadalupe Hernández; Servicio de Publicaciones, Universidad de la Rioja: Logroño, Spain, 2001; pp. 77-83.

9. De Jesus, M.N.; Petronilho, J. Sobolev orthogonal polynomials and $(M, N)$ coherent pairs of measures. J. Comput. Appl. Math. 2013, 237, 83-101. [CrossRef]

10. De Jesus, M.N.; Marcellán, F.; Petronilho, J.; Pinzón-Cortés, N.C. $(M, N)$-coherent pairs of order $(m, k)$ and Sobolev orthogonal polynomials. J. Comput. Appl. Math. 2014, 256, 16-35. [CrossRef]

11. Kwon, K.H.; Littlejohn, L.L. The orthogonality of the Laguerre polynomials $\left\{L_{n}^{(-k)}(x)\right\}$ for positive integers k. Ann. Numer. Math. 1995, 2, 289-303.

12. Pérez, T.E.; Piñar, M.A. On Sobolev orthogonality for the generalized Laguerre polynomials. J. Approx. Theory 1996, 86, 278-285. [CrossRef]

13. Álvarez de Morales, M.; Pérez, T.E.; Piñar, M.A. Sobolev orthogonality for the Gegenbauer polynomials $\left\{C_{n}^{(-N+1 / 2)}(x)\right\}_{n>0}$. J. Comput. Appl. Math. 1998, 100, 111-120. [CrossRef]

14. Alfaro, M.; Pérez, T.E.; Piñar, M.A.; Rezola, M.L. Sobolev orthogonal polynomials: The discrete-continuous case. Methods Appl. Anal. 1999, 6, 593-616. [CrossRef]

15. Alfaro, M.; Álvarez de Morales, M.; Rezola, M.L. Orthogonality of the Jacobi polynomials with negative integer parameters. J. Comput. Appl. Math. 2002, 145, 379-386. [CrossRef]

16. Sánchez-Lara, J.F. On the Sobolev orthogonality of classical orthogonal polynomials for non standard parameters. Rocky Mountain J. Math. 2017, 47, 267-288. [CrossRef]

17. López, G.; Marcellán, F.; Van Assche, W. Relative asymptotics for polynomials orthogonal with respect to a discrete Sobolev inner product. Constr. Approx. 1995, 11, 107-137. [CrossRef]

18. Marcellán, F.; Alfaro, M.; Rezola, M.L. Orthogonal polynomials on Sobolev spaces: Old and new directions. J. Comput. Appl. Math. 1993, 48, 113-131. [CrossRef]

19. Meijer, H.G. A short history of orthogonal polynomials in a Sobolev space. I. The non-discrete case. Nieuw Arch. Wisk. 1996, 14, 93-112.

20. Martínez-Finkelshtein, A. Asymptotic properties of Sobolev orthogonal polynomials. J. Comput. Appl. Math. 1998, 99, 491-510. [CrossRef]

21. Martínez-Finkelshtein, A. Analytic aspects of Sobolev orthogonal polynomials revisited. J. Comput. Appl. Math. 2001, 127, 255-266. [CrossRef]

22. Marcellán, F.; Moreno-Balcázar, J.J. Asymptotics and zeros of Sobolev orthogonal polynomials on unbounded supports. Acta Appl. Math. 2006, 94, 163-192. [CrossRef]

23. Marcellán, F.; Moreno-Balcázar, J.J. What is. . a Sobolev orthogonal polynomial? Notices Am. Math. Soc. 2017, 64, 873-875. [CrossRef]

24. Behr, N.; Dattoli, G.; Duchamp, G.H.E.; Licciardi, S.; Penson, K. A. Operational Methods in the Study of Sobolev-Jacobi Polynomials. Mathematics 2019, 7, 124. [CrossRef]

25. Ren, Y.; Yu, X.; Wang, Z. Diagonalized Chebyshev Rational Spectral Methods for Second-Order Elliptic Problems on Unbounded Domains. Numer. Math. Theor. Meth. Appl. 2019, 12, 265-284. [CrossRef]

26. Sharapudinov, I.I. Sobolev-orthogonal systems of functions and the Cauchy problem for ODEs. Izv. Ross. Akad. Nauk Ser. Mat. 2019, 83, 204-226; translation in Izv. Math. 2019, 83, 391-412. [CrossRef]

27. Sharapudinov, I.I. Sobolev-orthogonal systems of functions and some of their applications. Uspekhi Mat. Nauk 2019, 74, 87-164. (In Russian)

28. Mañas-Mañas, J.F.; Moreno-Balcázar, J.J. Classical Sobolev Orthogonal Polynomials: Eigenvalue Problem. Results Math. 2019, 74, 144. [CrossRef]

29. Bavinck, H. Differential and difference operators having orthogonal polynomials with two linear perturbations as eigenfunctions. J. Comput. Appl. Math. 1998, 92, 85-95. [CrossRef]

30. Welman, R. Regularized kernel learning in Hilbert scales. Unpublish work. 
31. Littlejohn, L.L.; Mañas-Mañas, J.F.; Moreno-Balcázar, J.J.; Wellman, R. Differential operator for discrete Gegenbauer-Sobolev orthogonal polynomials: Eigenvalues and asymptotics. J. Approx. Theory 2018, 230, 32-49. [CrossRef]

32. Szegő, G. Orthogonal Polynomials. In American Mathematical Society Colloquium Publications, 4th ed.; American Mathematical Society: Providence, RI, USA, 1975; Volume 23.

33. Mañas-Mañas, J.F.; Marcellán, F.; Moreno-Balcázar, J.J. Asymptotic behavior of varying discrete Jacobi-Sobolev orthogonal polynomials. J. Comput. Appl. Math. 2016, 300, 341-353. [CrossRef]

34. Askey, R.A.; Roy, R. Gamma function. In NIST Handbook of Mathematical Functions; Cambridge University Press: Cambridge, UK, 2010; pp. 135-147.

35. Arvesú, J.; Álvarez-Nodarse, R.; Marcellán, F.; Pan, K. Jacobi-Sobolev-type orthogonal polynomials: second-order differential equation and zeros. J. Comput. Appl. Math. 1998, 90, 135-156. [CrossRef]

36. Mañas-Mañas, J.F.; Marcellán, F.; Moreno-Balcázar, J.J. Asymptotics for varying discrete Sobolev orthogonal polynomials. Appl. Math. Comput. 2017, 314, 65-79. [CrossRef]

37. Marcellán, F.; Ronveaux, A. On a class of polynomials orthogonal with respect to a discrete Sobolev inner product. Indag. Math. 1990, 1, 451-464. [CrossRef]

(C) 2020 by the authors. Licensee MDPI, Basel, Switzerland. This article is an open access article distributed under the terms and conditions of the Creative Commons Attribution (CC BY) license (http://creativecommons.org/licenses/by/4.0/). 